\title{
RUPTURE AND DISSECTION IN PULMONARY ARTERY ANEURYSMS: INCIDENCE, CAUSE, AND TREATMENT—REVIEW AND CASE REPORT
}

Ömer Senbaklavaci, MD, ${ }^{\text {a }}$ Yukihiro Kaneko, MD, PhD, ${ }^{a}$ Anna Bartunek, MD, ${ }^{\mathrm{b}}$ Christof Brunner, MD, ${ }^{\mathrm{c}}$ Erol Kurkciyan, MD, ${ }^{\mathrm{d}}$

Patrick Wunderbaldinger, MD, ${ }^{\mathrm{e}}$ Walter Klepetko, MD, ${ }^{\mathrm{a}}$ Ernst Wolner, MD, ${ }^{\mathrm{a}}$ and Werner Mohl, MD, PhD, ${ }^{\mathrm{a}}$ Vienna, Austria

Since the first report of dissecting aneurysm of the pulmonary artery by Walshe in 1862 (cited by Watson ${ }^{1}$ ), 48 cases of pulmonary artery dissection have been reported. ${ }^{2-4}$ Dissection mostly develops in a pulmonary artery aneurysm associated with pulmonary hypertension and/or connective tissue disease. Forty-three cases were reported after death, whereas only 5 were diagnosed clinically, indicating that the event is highly lethal.

In this report, we describe the surgical repair of dissection of an aneurysm in the main pulmonary artery resulting from primary pulmonary hypertension.

Clinical summary. A 34-year-old woman was admitted to the emergency room with chest pain and shortness of breath. She had primary pulmonary hypertension for 10 years and had been receiving long-term therapy with coumarin and a

From the University of Vienna, Departments of Cardiothoracic Surgery, ${ }^{\mathrm{a}}$ Anesthesiology, ${ }^{\mathrm{b}} \quad$ Pulmonology, ${ }^{\mathrm{c}}$ Emergency Medicine, ${ }^{\mathrm{d}}$ and Radiology, ${ }^{\mathrm{e}}$ Vienna, Austria.

J Thorac Cardiovasc Surg 2001;121:1006-8

Copyright (C) 2001 by The American Association for Thoracic Surgery

$0022-5223 / 2001 \$ 35.00+0 \quad \mathbf{1 2 / 5 4 / 1 1 2 6 3 4}$

doi:10.1067/mtc.2001.112634 continuous intravenous infusion of epoprostenol (prostacyclin) for 4 years, with the dosage increasing to $16 \mathrm{ng} \cdot \mathrm{kg}^{-1}$. $\min ^{-1}$. Seven months previously, she underwent a right heart catheterization that revealed a pulmonary-systemic arterial pressure ratio $(\mathrm{Pp} / \mathrm{Ps})$ of 0.53 . The electrocardiogram showed right ventricular hypertrophy. A posteroanterior chest $\mathrm{x}$-ray film showed a left hilar mass indicating dilation of the left pulmonary artery. The patient was discharged without any further investigations because the physical examination disclosed no abnormalities. The next day, the patient was readmitted to the hospital because of worsening symptoms. The transthoracic echocardiogram revealed an enlarged main pulmonary artery with a diameter of $8 \mathrm{~cm}$, a competent pulmonary valve, and massive pericardial effusion but failed to demonstrate pulmonary artery dissection. A computed tomogram confirmed dissection of the main pulmonary artery (Fig $1, A)$. She was transferred to the operating theater with signs of imminent cardiac tamponade and shock. Suprasystemic pulmonary pressures (systolic $120 \mathrm{~mm} \mathrm{Hg}$ ) were noted after induction of anesthesia with a $\mathrm{Pp} / \mathrm{Ps}$ of 1.9.

Surgical technique. Femoro-femoral bypass was established urgently followed by sternotomy and pericardiotomy, which revealed massive bloody pericardial effusion. At a blood temperature of $32^{\circ} \mathrm{C}$ with the heart beating, the pulmonary 
Volume 121, Number 5

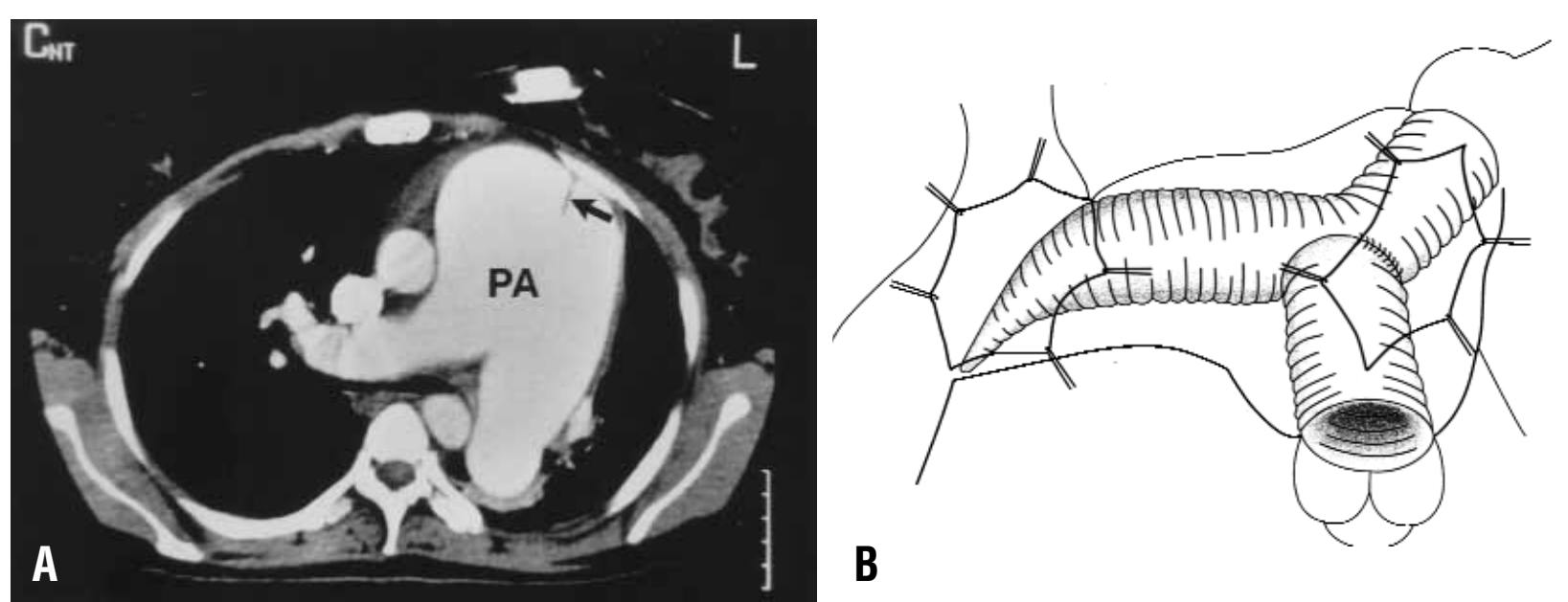

Fig 1. A, Chest computed tomogram shows dilated main pulmonary artery (PA), within which intimal flap (arrow) can be seen. B, Surgical procedure. T-shaped prosthetic graft replacement of the central pulmonary artery was performed by the inclusion technique. The pulmonary artery incision was closed to eliminate postoperative bleeding.

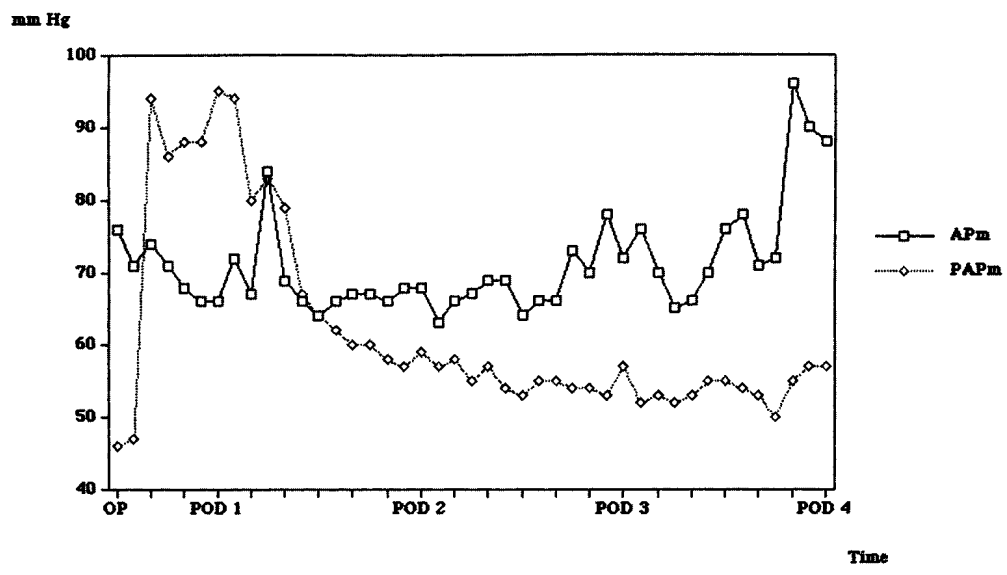

Fig 2. Postoperative systemic and pulmonary artery pressure change. Pulmonary artery pressure exceeded systemic pressure on postoperative $(P O D)$ day 1 . It gradually decreased in the following 2 days. $A P m$, Mean systemic arterial pressure; $P A P m$, mean pulmonary artery pressure.

artery was longitudinally incised from the sinotubular junction into the left pulmonary artery up to the left pericardial reflection. The right pulmonary artery incision was made separately between the aorta and superior vena cava. The pulmonary artery dissection was found spreading from the sinotubular junction up to the pericardial reflection on the left and the intermediate trunk on the right. An intimal tear was located between the main pulmonary artery and the intermediate trunk of the right pulmonary artery. The pulmonary valve appeared normal and competent. The torn intima at the intermediate trunk was repaired with 4-0 Prolene running suture (Ethicon, Inc, Somerville, NJ). The beveled end of a 32-mm tube graft was anastomosed end to side to the hilar right pulmonary artery with 5-0 Prolene running suture so that a T-shaped replacement of the central pulmonary artery could be done by the inclusion technique (Fig 1, B). The graft was then pulled through within the central right pulmonary artery to the left side. The free end of the graft was anastomosed in the same way to the hilar left pulmonary artery. Another 32-mm tube graft was interposed between the graft and sinotubular junction with 5-0 Prolene running suture. Finally, the pulmonary artery incision was closed with 4-0 Prolene running suture, burying the T-shaped graft within the pulmonary artery. After cardiopulmonary bypass was discontinued, the patient again had suprasystemic pulmonary pressure. Continuous infusion of alprostadil (prostaglandin $\mathrm{E}_{1}$ ) at $0.2 \mu \mathrm{g} \cdot \mathrm{kg}^{-1} \cdot \mathrm{min}^{-1}$ and nitric oxide inhalation with $8 \mathrm{ppm}$ was administered before the patient was transferred to the intensive care unit. 
On the first postoperative day, alprostadil infusion was changed to epoprostenol (prostaglandin $\mathrm{I}_{2}$ ) at dosages of 16 to $12 \mu \mathrm{g} \cdot \mathrm{kg}^{-1} \cdot \mathrm{min}^{-1}$ and milrinone of 0.4 to $0.2 \mu \mathrm{g} \cdot \mathrm{kg}^{-1}$. $\mathrm{min}^{-1}$. Hemofiltration was performed from postoperative day 1 for 48 hours because of oliguric renal insufficiency. Severe pulmonary hypertension improved gradually with decrement of Pp/Ps from 1.6 to 0.7 (Fig 2).

The fluid requirements decreased dramatically on the third postoperative day with recovery of renal function and decrease in blood lactate concentration. The following course was uneventful. The patient was weaned from nitric oxide inhalation on the sixth postoperative day and was extubated on the eleventh postoperative day. On the thirteenth postoperative day, the patient was transferred to the general ward with still elevated but subsystemic pulmonary pressures $(\mathrm{Pp} / \mathrm{Ps}=0.6)$ receiving epoprostenol, $16 \mathrm{ng} \cdot \mathrm{kg}^{-1} \cdot \mathrm{min}^{-1}$. The patient was discharged on postoperative day 45 .

A postoperative computed tomogram showed a sealed leak around the distal tube graft covered with the native pulmonary artery. Pathologic examination of the pulmonary arterial wall showed myxoid degeneration of the endothelial surface and confirmed the presence of dissection within the aneurysmal wall. The histologic examination showed that the specimen did not contain collagen type I or type III.

Because of persistent pulmonary hypertension, the patient was accepted for lung transplantation. She underwent double lung transplantation with the use of an extracorporal membrane oxygenator 9 months after the emergency operation. Intraoperatively, the sealed leakage was found to be thrombosed. The pulmonary prosthesis was replaced, with a short part of the prosthesis being left in place connecting the main pulmonary artery with the transplants. The patient was discharged 3 weeks after the operation in good condition and remained well 10 months after the transplantation.

Discussion. Dissection of the pulmonary artery is a rare but life-threatening event. Although the pathophysiologic cause of dissection in pulmonary artery aneurysm is not clear, pulmonary hypertension and resultant mucoid degeneration of the media and fragmentation of the elastic fibers strongly predispose to this condition. In the literature, dissection of the pulmonary artery always occurs at the site of a pulmonary aneurysm or dilation. It is reasonable to assume that pulmonary artery dissection occurs at the point where pulmonary artery tissue becomes too fragile to support the tension of the pulmonary artery wall. Tissue fragility is caused by infectious and connective tissue diseases, and high wall tension is caused by high internal pressure and a large-radius aneurysm, as predicted by LaPlace's law. In pulmonary artery dissection, the false lumen tends to rupture rather than to develop a re-entry site, as is usual in aortic dissection. Six cases of pulmonary artery dissection associated with primary pulmonary hypertension have previously been reported.
$\mathrm{Yu}$ and colleagues ${ }^{5}$ showed that epoprostenol exerts inhibitory actions on proliferation and DNA synthesis of rat cardiac fibroblasts and messenger RNA expression for collagen types I and III. In our patient, long-term continuous epoprostenol infusion in the presence of severe pulmonary hypertension might have predisposed to aneurysmal change and subsequent biochemical changes within the vessel wall of the pulmonary artery leading to dissection.

The symptoms of pulmonary artery dissection are nonspecific, with $82 \%$ of patients having exertional dyspnea, $67 \%$ retrosternal chest pain, and 52\% central cyanosis. The diagnosis was made by echocardiography, computed tomography, and/or magnetic resonance imaging. Patients with pulmonary hypertension and those receiving long-term epoprostenol infusion having these symptoms should be examined urgently with echocardiography and computed tomography to exclude pulmonary artery dissection. In our patient, the computed tomogram should have been obtained on the first admission because of her primary pulmonary hypertension.

Because pulmonary artery dissection is highly lifethreatening, we believe patients with pulmonary artery dissection should undergo emergency surgery to prevent lethal bleeding. However, the literature contains only one report of a patient with dissection of the pulmonary artery undergoing surgery. That patient had chronic dissection of the pulmonary artery without pulmonary hypertension. To our knowledge, we are reporting on the first successful treatment of a patient who underwent surgical repair of acute pulmonary artery dissection.

Histologic examination was done by Prof Dr M. Susani from the Pathology Department of the University of Vienna.

Received for publication Aug 24, 2000; accepted for publication Oct 24, 2000.

Address for reprints: Werner Mohl, MD, University of Vienna, Department of Cardiothoracic Surgery, Währinger Gürtel 18-20, A-1090 Vienna, Austria (E-mail: werner.mohl@univie.ac.at).

\section{REFERENCES}

1. Watson AJ. Dissecting aneurysm of arteries other than the aorta. J Pathol Bacteriol 1956;72:439-49.

2. Luctrath H. Dissecting aneurysm of the pulmonary artery. Virchows Arch [Pathol Anat] 1981;391:241-7.

3. Walley VM, Virmani RV, Silver MD. Pulmonary arterial dissections and ruptures: to be considered in patients with pulmonary arterial hypertension presenting with cardiogenic shock or sudden death. Pathology 1990;22:1-4.

4. Steurer J, Jenni R, Medici TC, Vollrath T, Hess OM, Segenthaler W. Dissecting aneurysm of the pulmonary artery with pulmonary hypertension. Am Rev Respir Dis 1990;142:1219-21.

5. Yu H, Gallagher AM, Garfin PM, Printz MP. Prostacyclin release by rat cardiac fibroblasts: inhibition of collagen expression. Hypertension 1997;30:1047-53. 